\title{
Preferential depletion of gut CD4-expressing iNKT cells contributes to systemic immune activation in HIV-1 infection
}

\author{
FJ Ibarrondo ${ }^{1}$, SB Wilson ${ }^{2}$, LE Hultin ${ }^{1}$, R Shih ${ }^{1}$, MA Hausner ${ }^{1}$, PM Hultin $^{1}$, PA Anton ${ }^{1}$, BD Jamieson ${ }^{1}$ and \\ OO Yang ${ }^{1,3}$
}

Chronic inappropriate immune activation is the central defect-driving loss of CD4 ${ }^{+} \mathrm{T}$ helper cells and progression to AIDS in persons with HIV-1 infection, but the mechanisms remain controversial. We examined key regulatory invariant receptor natural killer T (iNKT) cells in the gut, the largest reservoir of lymphocytes and a key arena of HIV-1 pathogenesis. In healthy control persons, the anti-inflammatory CD4 ${ }^{+}$iNKT-cell subset predominated over the proinflammatory CD4 ${ }^{-}$iNKT-cell subset in the gut, but not in the blood, compartment. HIV-1 infection resulted in a preferential loss of this anti-inflammatory CD4 ${ }^{+}$iNKT-cell subset within the gut. The degree of loss of the CD4 ${ }^{+}$iNKT-cell subset in the gut, but not in the blood, correlated to the systemic immune activation and exhaustion that have been linked to disease progression. These results suggest a potentially important contribution of gut iNKT-cell imbalance in determining the systemic immune activation that is the hallmark of HIV-1 pathogenesis.

\section{INTRODUCTION}

Invariant T-cell receptor "natural killer T cells" (iNKT-cells) are a T-cell subset characterized by the expression of a semiinvariant T-cell receptor (TCR) that is typically composed of paired V $\alpha 24$ and V $\beta 11$ chains. ${ }^{1,2}$ This TCR recognizes endogenous $^{3,4}$ and exogenous glycolipids presented by CD1d, a major histocompatibility complex-like molecule. ${ }^{2}$ It has been demonstrated that iNKT cells modulate adaptive immunity via interactions with myeloid dendritic cells, ${ }^{5}$ likely bridging innate and adaptive immunity through signalling lymphocyte activation molecule and cytokine receptors. ${ }^{2,3,6}$

Although iNKT cells are a rare subpopulation among $\mathrm{T}$ cells, they produce key immunomodulatory cytokines upon activation. ${ }^{3,7}$ The iNKT cells can be divided into two functional subsets determined by the expression of $\mathrm{CD} 4 .{ }^{1} \mathrm{CD} 4{ }^{+}$iNKT cells have a $\mathrm{T}$ helper type 0 (Th0) profile, generally produce both Th1 and Th2 cytokines upon activation, principally interferon gamma (IFN- $\gamma)$, tumor necrosis factor, interleukin (IL)-4, and IL-10, and are thought to be anti-inflammatory and pro-atopic. By contrast, $\mathrm{CD} 4^{-}$iNKT cells generally have a pure Th1 profile and are thought to be pro-inflammatory. ${ }^{8-10}$ Given that these iNKT-cell subsets are functionally distinct, they likely have key opposing roles in the modulation of adaptive immunity; imbalances between these subsets have been linked to autoimmune diseases. ${ }^{1,11}$

Activated memory $\mathrm{CD} 4{ }^{+} \mathrm{T}$ cells are the major reservoir for HIV-1 replication, as these cells express CCR5 and key transcription factors needed for efficient viral entry and transcription. $\mathrm{CD}^{+}{ }^{+}$iNKT cells have this activated memory phenotype with CCR 5 expression ${ }^{12-14}$ and have been proposed to be susceptible to HIV-1 infection. ${ }^{13,15-17}$ Blood levels of the $\mathrm{CD}^{+}{ }^{+}$subset of iNKT cells are depleted in persons with chronic HIV-1 infection, and this defect has been proposed to contribute to immune dysregulation. ${ }^{15,18-20}$

Gut-associated lymphoid tissue is the largest compartment of activated T cells in the body ${ }^{21-23}$ and is a key site for initial establishment of HIV-1 infection regardless of route of transmission, as well as being a reservoir for chronic infection. ${ }^{24,25}$ Abnormalities in the gut induced directly by HIV-1 infection have been suggested to disrupt immune

${ }^{1}$ Department of Medicine and UCLA AIDS Institute, David Geffen School of Medicine at University of California Los Angeles, Los Angeles, California, USA. ${ }^{2}$ Department of Pathology, Immunology, and Laboratory Medicine, University of Florida, Gainesville, Florida, USA and ${ }^{3}$ Department of Microbiology, Immunology, and Molecular Genetics, David Geffen School of Medicine at University of California Los Angeles, Los Angeles, California, USA. Correspondence: OO Yang (oyang@mednet.ucla.edu) 
containment of gut bacterial flora, leading to the systemic immune activation that drives disease progression. ${ }^{26,27}$ Given the proposed consequences of $\mathrm{CD} 4{ }^{+}$iNKT-cell depletion and abnormal gut immunity in $\mathrm{HIV}-1$ pathogenesis, we have examined the phenotypes and levels of iNKT-cell subsets in the gut and blood compartments of HIV-1-infected and uninfected individuals as potential correlates to the chronic immune activation that drives disease progression.

\section{RESULTS}

Sampling protocol and clinical status of study subjects

All subjects were confirmed by pre-screening to have quantifiable iNKT cells in the blood $(\geqslant 0.002 \%$ of peripheral blood mononuclear cells, Supplementary Figure S1 online) before enrollment; of the 27 pre-screened volunteers, two did not meet this threshold and were excluded. The enrolled participants were 25 Caucasian men, including 18 with chronic HIV-1 infection who were self-reported as untreated with antiviral medications and 7 control men who were HIV-1uninfected (Supplementary Table S1 online). Median ages were 45 years (range 24-63 years) for the HIV-1-infected group and 37 years (range 21-50 years) for the control group $(P=0.067)$. Viremia in the infected men ranged from $<50$ to 68,000 RNA copies per $\mathrm{ml}$ of plasma, and peripheral blood $\mathrm{CD}^{+}{ }^{+}$T-cell counts ranged from 196 to 911 cells per $\mu \mathrm{l}$. Each participant underwent two bi-weekly visits for phlebotomy and sigmoid colonic biopsies. Data from both visits were averaged.

\section{The gut mucosa normally is enriched for CD4 ${ }^{+} \mathrm{T}$ cells and is a pro-inflammatory environment compared with peripheral blood}

The T-lymphocyte populations in the peripheral blood and sigmoid gut mucosa were compared in the control HIV-1uninfected group (Figure 1). Overall, the gut mucosa was relatively higher in the percentage of $\mathrm{CD} 4{ }^{+} \mathrm{T}$ cells and lower in
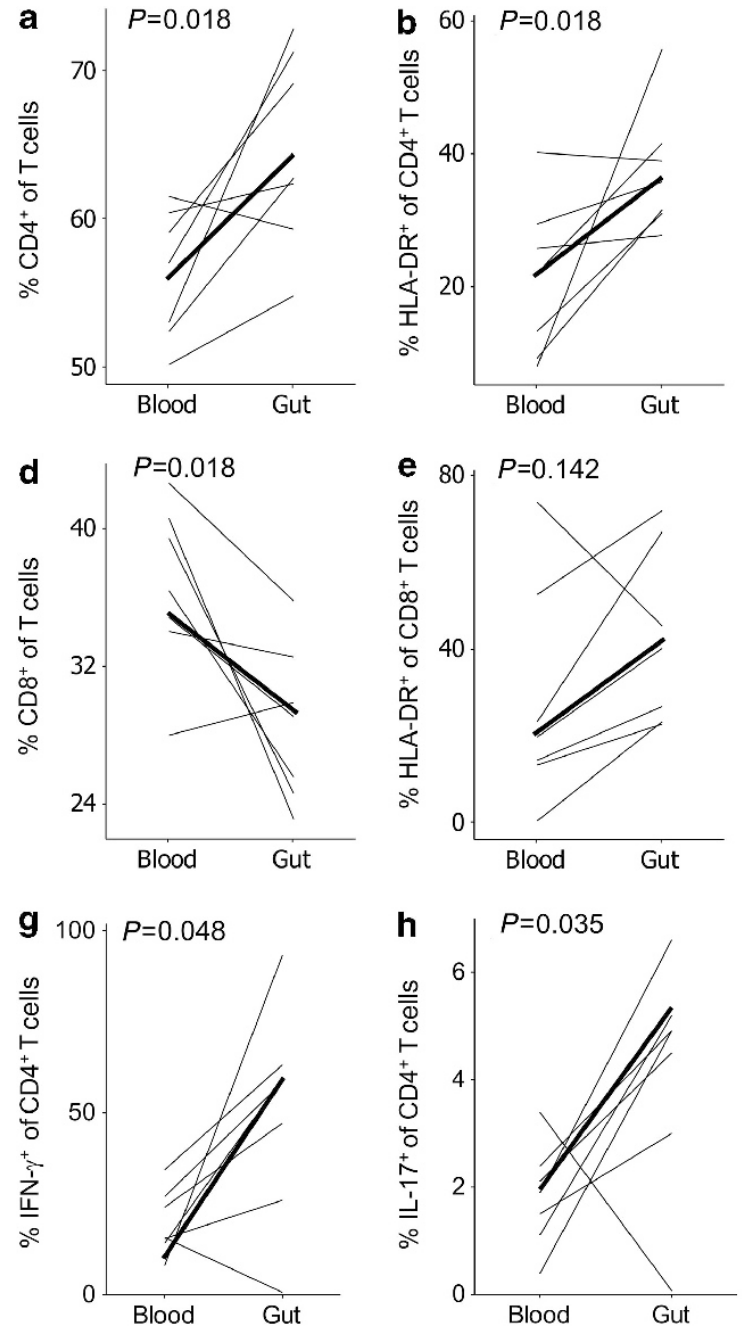
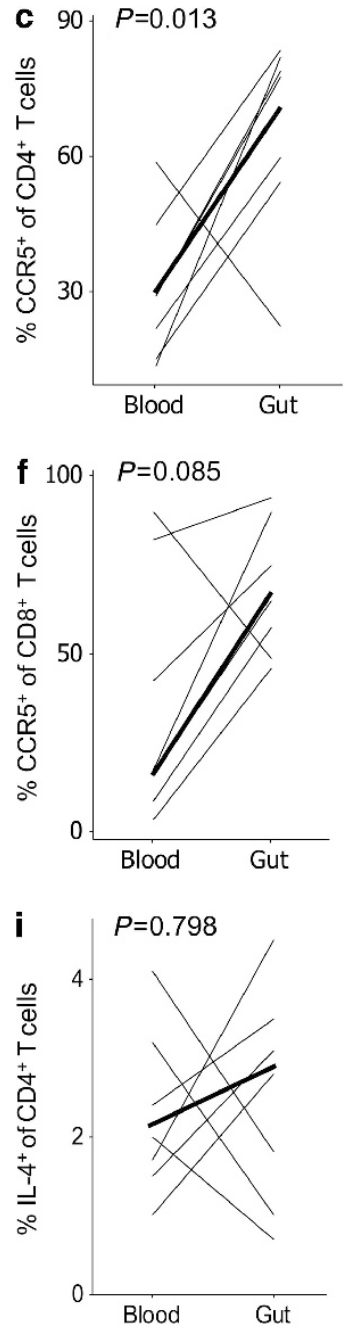

Figure 1 Frequencies, activation status, and functionality of bulk CD4 ${ }^{+}$and $\mathrm{CD} 8^{+} \mathrm{T}$ cells in the blood and gut mucosal compartments in healthy control individuals. (a-c) $\mathrm{CD}^{+}$and $(\mathbf{d}-\mathbf{f}) \mathrm{CD}^{+} \mathrm{T}$ cells are quantified and examined for expression of human leukocyte antigen (HLA)-DR and CCR5 in both the blood and gut compartments. (g-i) Cytokine production by CD4 ${ }^{+}$T cells from both the compartments after stimulation are plotted. Heavy lines represent the medians. IFN, interferon; IL, interleukin. 
the percentage of $\mathrm{CD} 8^{+} \mathrm{T}$ cells compared with the blood $\left(P=0.018\right.$, Figure 1a,d). Evaluation of $\mathrm{CD} 4^{+} \mathrm{T}$-cell activation revealed significantly increased frequencies of human leukocyte antigen (HLA)-DR and CCR5 expression on $\mathrm{CD} 4{ }^{+} \mathrm{T}$ cells in the gut vs. blood compartments $(P=0.018$ and $P=0.013$, Figure $\mathbf{1 b}, \mathbf{c})$, with similar (but not statistically significant) trends observed for $\mathrm{CD}^{+}{ }^{+} \mathrm{T}$ cells $(P=0.142$ and $P=0.085$, Figure 1e,f), demonstrating greater immune activation in the gut compartment. Evaluation of the cytokine profiles of CD4 ${ }^{+}$ $\mathrm{T}$ cells revealed significantly higher capacities to produce IFN $-\gamma$ and IL-17 in the gut compared with blood $(P=0.048$ and $P=0.035$, Figure $\mathbf{1 g}, \mathbf{h}$ ), additionally demonstrating a more pro-inflammatory cytokine milieu in the gut compartment. There was no consistent pattern for IL-4 production by gut vs. blood $\mathrm{CD} 4^{+} \mathrm{T}$ cells (Figure 1i). Overall, these data confirm that the gut is normally a highly immune-activated proinflammatory environment, in agreement with previous reports.
The gut mucosa normally is enriched for activated $\mathrm{CD} 4^{+}$ iNKT cells compared with peripheral blood

Regulatory iNKT cells were assessed in the gut and blood compartments of the control persons. Within the total $\mathrm{CD}^{+}$ T-cell population, there were higher frequencies of total iNKT cells in the gut compared with the blood in six of the seven subjects, although this trend was insignificant due to the outlier (Figure 2a). Examining $\mathrm{CD} 4^{+}$and $\mathrm{CD} 4^{-}$iNKT-cell subsets, $\mathrm{CD} 4{ }^{+}$iNKT cells were more frequent in the gut compared with blood $(P=0.048$, Figure $2 \mathbf{b})$, while the $\mathrm{CD}^{-}$subset was similar between the compartments (Figure 2c). Activation marker analysis of total iNKT cells in both the compartments demonstrated significantly higher percentages of HLA-DR $(P=0.018$, Figure 2d) on the total iNKT population in the gut compared with blood. Percentages of total iNKT cells expressing CCR5 were higher in the gut for five of the seven subjects, although not statistically significant $(P=0.085$, Figure $\mathbf{2 g}$ ). These differences in activation were more
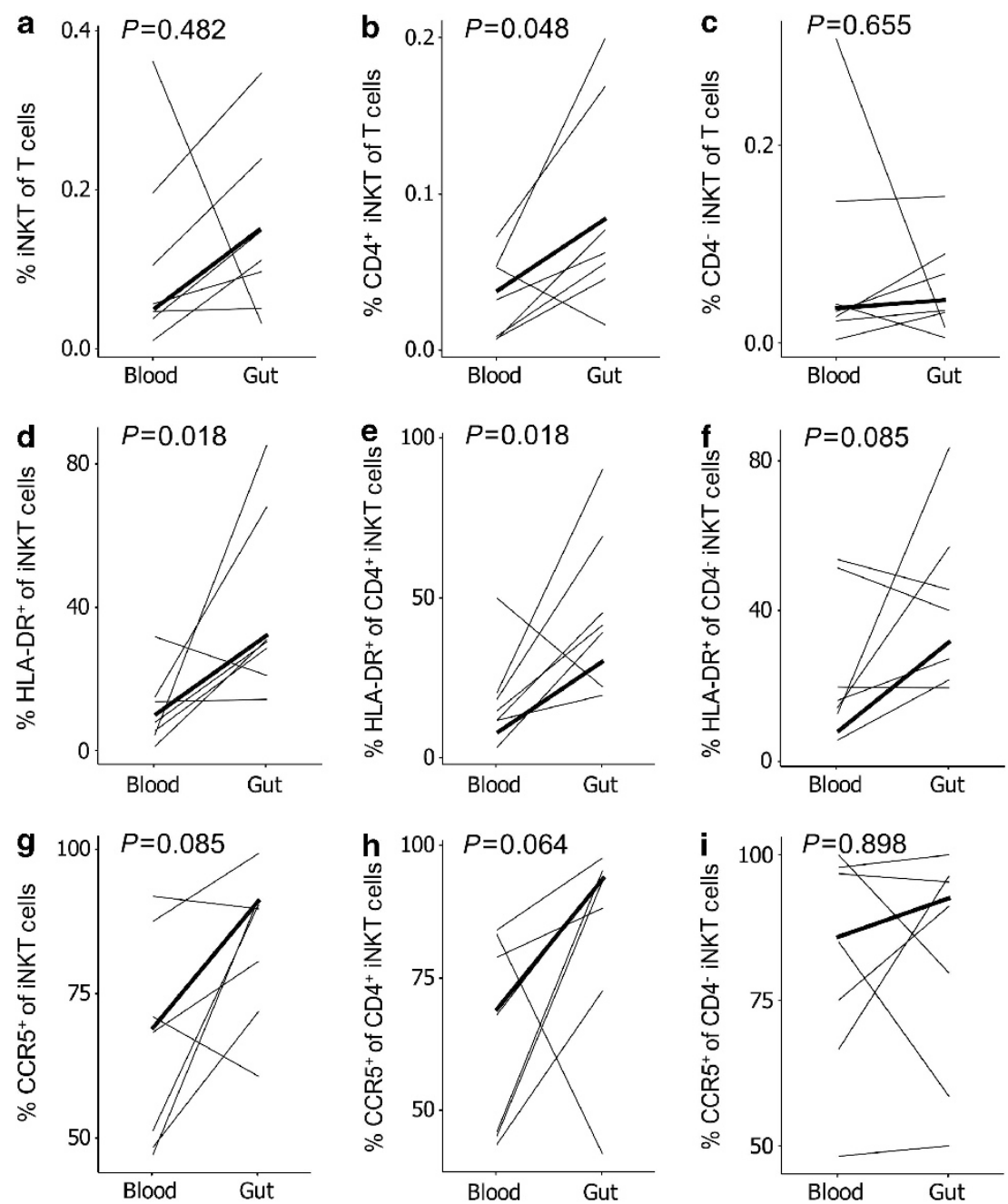

Figure 2 Frequencies of iNKT-cell (invariant natural killer T cell) subsets in the blood and gut mucosal compartments of healthy control individuals. (a-c) The concentrations of total, $\mathrm{CD} 4^{+}$, and $\mathrm{CD} 4^{-}$iNKT cells as a percentage of all $\mathrm{CD} 3^{+} \mathrm{T}$ cells in blood and gut mucosal compartments are plotted. Percentages of each of these populations expressing (d-f) human leukocyte antigen (HLA)-DR and ( $\mathbf{g}-\mathbf{i})$ CCR5 are plotted. Heavy lines indicate the medians. 
consistent in the iNKT-cell $\mathrm{CD}^{+}$subset $(P=0.018$ and $P=0.064$, Figure 2e,h) compared with the $\mathrm{CD}^{-}$subset $(P=0.085$ and $P=0.898$, Figure $2 \mathbf{f}, \mathbf{i})$. Analysis of IL-4 production confirmed that a higher percentage of $\mathrm{CD}^{+}$ iNKT cells produced IL-4 compared with the general CD4 ${ }^{+} \mathrm{T}$ lymphocyte population (data not shown). Altogether, these results suggest that increased frequency and activation of $\mathrm{CD}^{+}{ }^{+}$iNKT cells have a counterbalancing role in the inflammatory milieu of the gut.

\section{HIV-1 infection causes relative reduction of total activated $\mathrm{CD}^{+}{ }^{+} \mathrm{T}$ cells and increase of total activated CD8 ${ }^{+}$ $\mathrm{T}$ cells in both the peripheral blood and gut mucosal compartments}

Percentages of $\mathrm{CD} 4{ }^{+}$and $\mathrm{CD} 8{ }^{+}$subsets among all the T cells were assessed in persons with HIV-1 infection compared with uninfected control subjects. As expected, the $\mathrm{CD}^{+}{ }^{+} \mathrm{T}$-cell percentage was lower in the infected group compared with the control group for both the gut and blood compartments (Figure 3a). This reduction was particularly marked for the CCR5-expressing subset vs. the HLA-DR-expressing subset in both the compartments (Figure 3b,c), consistent with the role of CCR5 as a co-receptor for HIV-1. The opposite pattern was seen for the $\mathrm{CD}^{+}{ }^{+}$T-cell population, which demonstrated particularly increased activation in the gut compartment (Figure 3d-f). These findings confirm the effect of HIV-1 infection depleting the activated and susceptible CD4 ${ }^{+} \mathrm{T}$ cells in both the blood and gut compartments and driving systemic immune activation.
HIV-1 infection causes preferential loss of activated CD4 ${ }^{+}$ iNKT cells in the gut compartment

The concentrations of iNKT cells as percentages of total T cells in the gut and blood compartments were compared between the HIV-1-infected and control groups (Figure 4). In the HIV1-infected individuals, iNKT cells in both the compartments were not statistically different (Figure 4a), while the balance of $\mathrm{CD} 4{ }^{+}$to $\mathrm{CD} 4{ }^{-}$subsets of iNKT cells was skewed significantly in the gut but not in the blood $(P=0.040$ vs. $P=1.000$, Figure $4 \mathbf{b}$ ). This altered balance was mediated predominately by loss of the $\mathrm{CD} 4{ }^{+}$iNKT population, which was significantly depleted in the gut but not in the blood $(P=0.005$ vs. $P=0.065$, Figure 4c). The same pattern of preferential depletion in the gut was seen when quantifying the $\mathrm{CCR}^{+}{ }^{+}$and HLA-DR ${ }^{+}$subsets of these cells $(P=0.004-$ 0.028 , Figure 4d,e). By contrast, the CD4 ${ }^{-}$iNKT-cell subset was similar in frequency for HIV-1-infected and uninfected persons in both the compartments (Figure $\mathbf{4 f - h}$ ). In agreement with the susceptibility of $\mathrm{CD}^{+}{ }^{+}$iNKT cells to HIV-1 infection, ${ }^{15,18,28}$ these data demonstrate preferential depletion of the anti-inflammatory $\mathrm{CD} 4{ }^{+}$iNKT cells in the gut compartment of HIV-1-infected persons, suggesting dysregulation in this pivotal immune reservoir.

\section{Regulatory cytokine production by total CD4 ${ }^{+} \mathrm{T}$ cells and iNKT-cell subsets are differentially affected by HIV-1 infection in the blood vs. gut compartments}

The effects of HIV-1 infection on the capability of total CD4 ${ }^{+}$ $\mathrm{T}$ cells and iNKT-cell subsets to produce regulatory cytokines


Figure 3 Concentrations and activation of total $\mathrm{CD} 4^{+}$and $\mathrm{CD} 8^{+} \mathrm{T}$ cells in the blood and gut mucosal compartments in persons with and without $\mathrm{HIV}-1$ infection. The concentrations and expression of CCR5 and human leukocyte antigen (HLA)-DR are plotted for (a-c) CD4 ${ }^{+}$and (d-f) CD8 ${ }^{+} \mathrm{T}$ cells in both the blood and gut mucosal compartments of persons with (gray boxes) and without (white boxes) HIV-1 infection are plotted. 
a

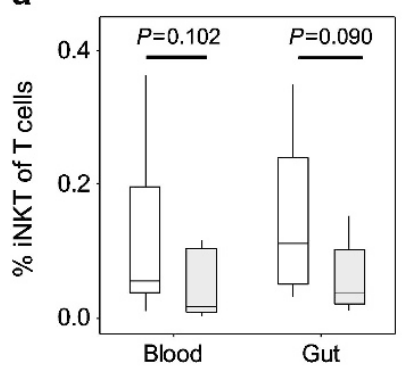

c

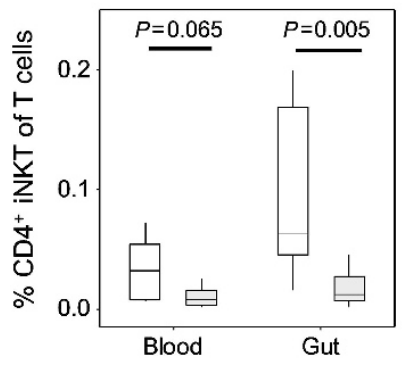

f

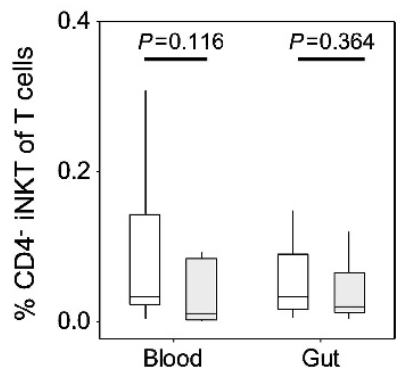

b

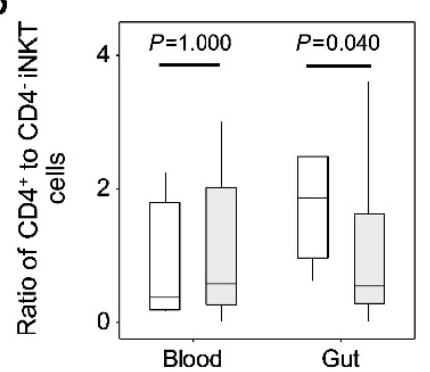

d

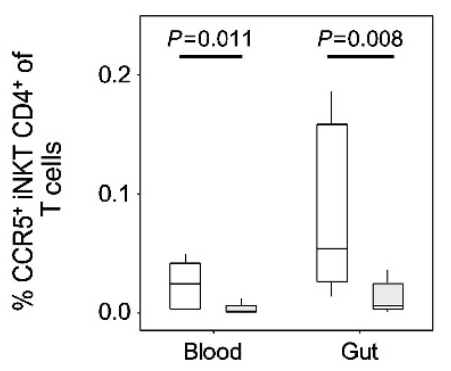

g

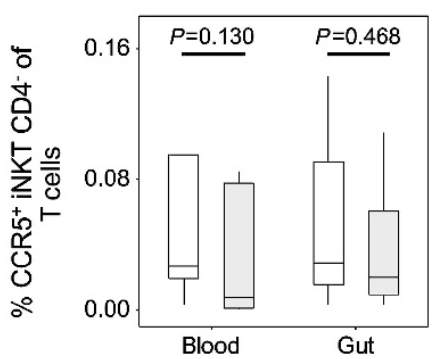

$\square$ Uninfected control subjects

HIV-1-infected subjects



h

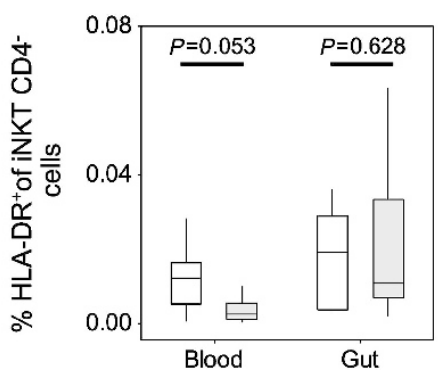

Figure 4 Concentrations and activation of iNKT cells (invariant natural killer T cells) in the blood and gut mucosal compartments in persons with and without HIV-1 infection. (a) The concentrations of total iNKT cells in the blood and gut mucosal compartments are plotted, as well as (b) the ratios of the $\mathrm{CD}^{+}{ }^{+}$to $\mathrm{CD} 4^{-}$iNKT cells in each of these compartments. Concentrations and expression of CCR5 and human leukocyte antigen (HLA)-DR are plotted for the $(\mathbf{c}-\mathbf{e}) \mathrm{CD}^{+}$and $(\mathbf{f}-\mathbf{h}) \mathrm{CD} 4^{-}$iNKT-cell subsets. Persons with (gray boxes) and without (white boxes) HIV-1 infection are compared.

were examined in the blood and gut compartments (Figure 5). As percentages of the total T-cell population, there was preservation of IFN- $\gamma$-producing total $\mathrm{CD}^{+}{ }^{+} \mathrm{T}$ cells and iNKT-cell subsets in both the compartments (Figure 5a-c). HIV-1 infection was associated with a net increase of IL-4producing total $\mathrm{CD} 4{ }^{+} \mathrm{T}$ cells in the gut but not in the blood $(P=0.040$ and $P=0.188$, Figure 5d). There was reduction of IL-4-producing $\mathrm{CD} 4{ }^{+}$iNKT cells in the blood with a potential trend for increase in the gut $(P=0.020$ and $P=0.220$, Figure 5e) but no appreciable change in IL-4 production by the $\mathrm{CD}^{-}$iNKT-cell population (Figure 5f). IL-17producing $\mathrm{CD} 4{ }^{+} \mathrm{T}$ cells were decreased in the blood and not significantly reduced in the gut $(P=0.030$ and $P=0.306$, Figure $5 \mathrm{~g}$ ), whereas there was undetectable production of this cytokine by either iNKT-cell subset (data not shown). Overall, these data demonstrate that Th1 pro-inflammatory function is preserved in the gut and blood after HIV-1 infection and that there may be an associated compensatory increase in Th2 activity in the gut in response to increased inflammation.
Activation and exhaustion of $\mathrm{CD}^{+} \mathrm{T}$ cells in the gut are correlates of immune containment of HIV-1

Peripheral blood $\mathrm{CD}^{+} \mathrm{T}$ cells have been found to exhibit correlations between activation or exhaustion with viremia, suggesting importance of the functional status of these cells in immune containment of infection. ${ }^{29}$ The activation and exhaustion of $\mathrm{CD} 8{ }^{+} \mathrm{T}$ cells in the gut and blood compartments was compared with plasma viremia levels in the HIV-1-infected persons. The percentage of CD8 ${ }^{+} \mathrm{T}$ cells expressing HLA-DR in either the gut or the blood were both positively correlated to viremia $(P=0.007$ and $P=0.006$, Figure 6a,d), while the percentage expressing programmed cell death- 1 in the gut was better correlated to viremia than that in the blood $(P=0.048$ and $P=0.110$, Figure 6b,e). Moreover, CD $4^{+}$iNKT-cell levels in the gut, but not in the blood, correlated negatively with viremia $(P=0.024$ and $P=0.169$, Figure $6 \mathbf{c}, \mathbf{f})$, implying loss of these regulatory cells. These observations suggest that the crucial interactions between cellular immunity and HIV-1 may occur in the gut compartment rather than in the peripheral blood. 

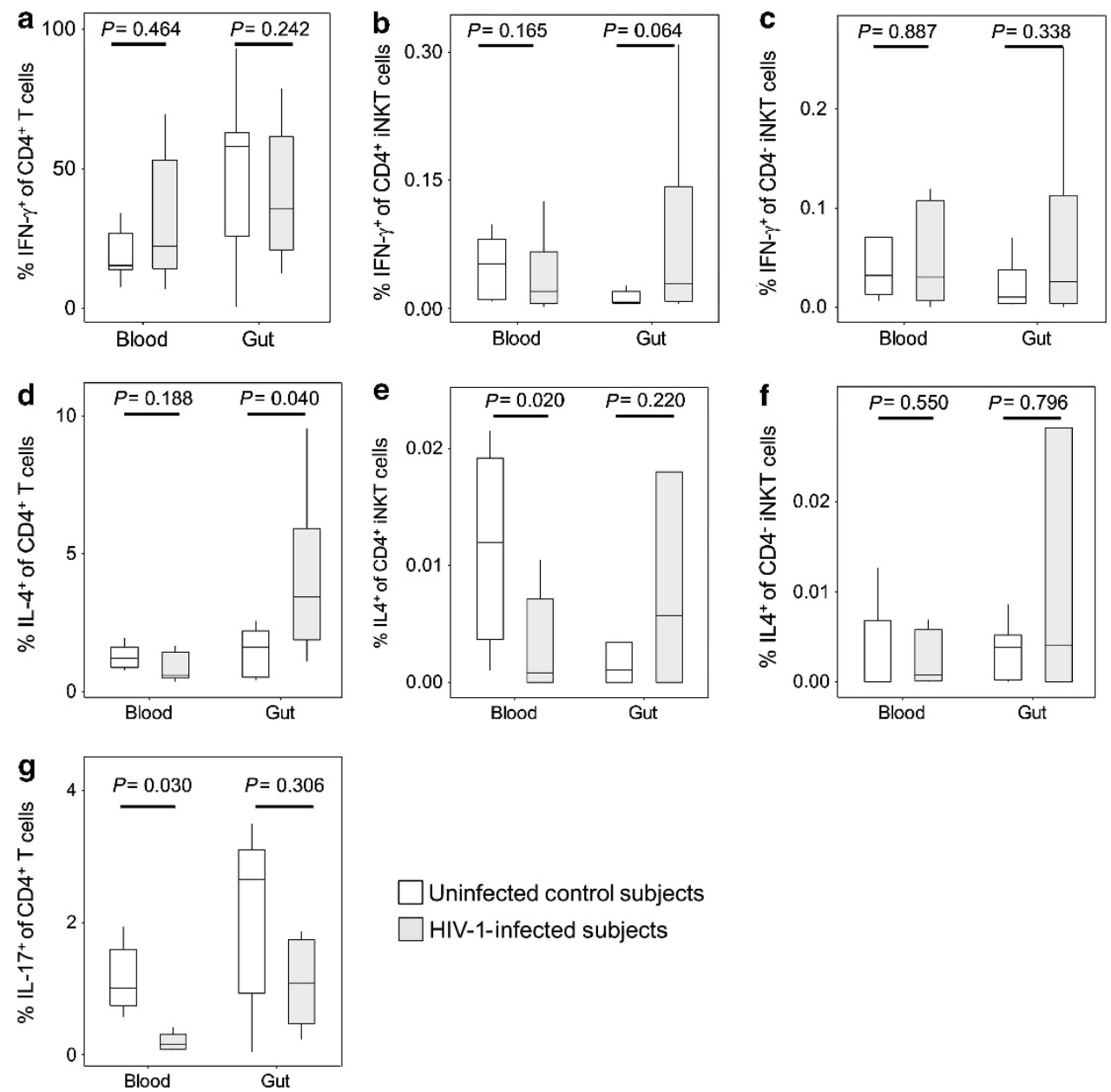

$\square$ Uninfected control subjects

HIV-1-infected subjects

Figure 5 Cytokine production profile of CD4 ${ }^{+}$T cells and iNKT-cell (invariant natural killer T cell) subsets in the blood and gut mucosal compartments in persons with and without HIV-1 infection. The concentrations of cells producing (a-c) interferon (IFN)- $\gamma$, (d-f) interleukin (IL)-4 and (g) IL-17 are indicated for total CD4 ${ }^{+}$T cells $(\mathbf{a}, \mathbf{d}, \mathbf{g}), \mathrm{CD}^{+}{ }^{+}$iNKT cells $(\mathbf{b}, \mathbf{e})$ and CD4 ${ }^{-}$iNKT cells $(\mathbf{c}, \mathbf{f})$ in the blood and gut mucosal compartments of persons with (gray boxes) and without (white boxes) HIV-1 infection.

The balance of CD4 ${ }^{+}$iNKT cells in the gut determines the general state of immune activation in HIV-1 infection

Because $\mathrm{CD}^{+}$and $\mathrm{CD}^{-}{ }^{-}$iNKT-cell subpopulations are believed to have opposing roles in modulation of inflammation, we compared the balance (ratios) of these iNKT-cell subpopulations with activation and exhaustion of $\mathrm{CD} 8^{+} \mathrm{T}$ cells in the HIV-1-infected persons. The $\mathrm{CD}^{+}{ }^{+}$to $\mathrm{CD} 4^{-}$ratio of iNKT cells in the gut correlated significantly to CD8 ${ }^{+}$T-cell activation and exhaustion in the gut $(P=0.037$ and $P=0.032$, Figure 6g,h) and blood $(P=0.033$ and $P=0.054$, Figure 6i,j). By contrast, the ratio in the blood did not correlate to these $\mathrm{CD}^{+}{ }^{+} \mathrm{T}$-cell parameters in either compartment (Figure 6k-n). These data suggest that HIV-1-mediated iNKT perturbation in the gut rather than in the blood is a crucial contributor to the systemic immune activation that is the hallmark of HIV-1 pathogenesis.

\section{DISCUSSION}

This study is the first reported examination of iNKT-cell frequency, phenotype, and function in the gut lymphocyte compartment of HIV-1-infected persons. These cells have been studied in a variety of autoimmune and infectious diseases and are believed to have important roles in modulating adaptive immunity. Although previous studies of iNKT cells in the blood have demonstrated their susceptibility to HIV-1 infection and depletion in persons with HIV-1 infection, ${ }^{15,17-19}$ correlation of this finding to the pathogenesis of HIV-1 infection has been lacking. Our current study provides evidence that HIV-1-induced depletion of $\mathrm{CD}^{+}{ }^{+}$iNKT cells in the gut (but not in the blood) compartment has a pivotal role in the inappropriate immune activation that is the hallmark of disease progression to AIDS.

Physiologically an inflammatory environment rich in activated memory $\mathrm{CD} 4{ }^{+} \mathrm{T}$ cells and home to approximately $60 \%$ of total body lymphocytes, ${ }^{21,23}$ the gastrointestinal tract has been implicated as the key venue where HIV-1 infection begins in acute infection and causes immune damage, leading to disease progression in chronic infection. ${ }^{30,31}$ It has been proposed that generalized $\mathrm{CD}_{4}{ }^{+} \mathrm{T}$-cell depletion in the gut during acute infection leads to ongoing microbial 

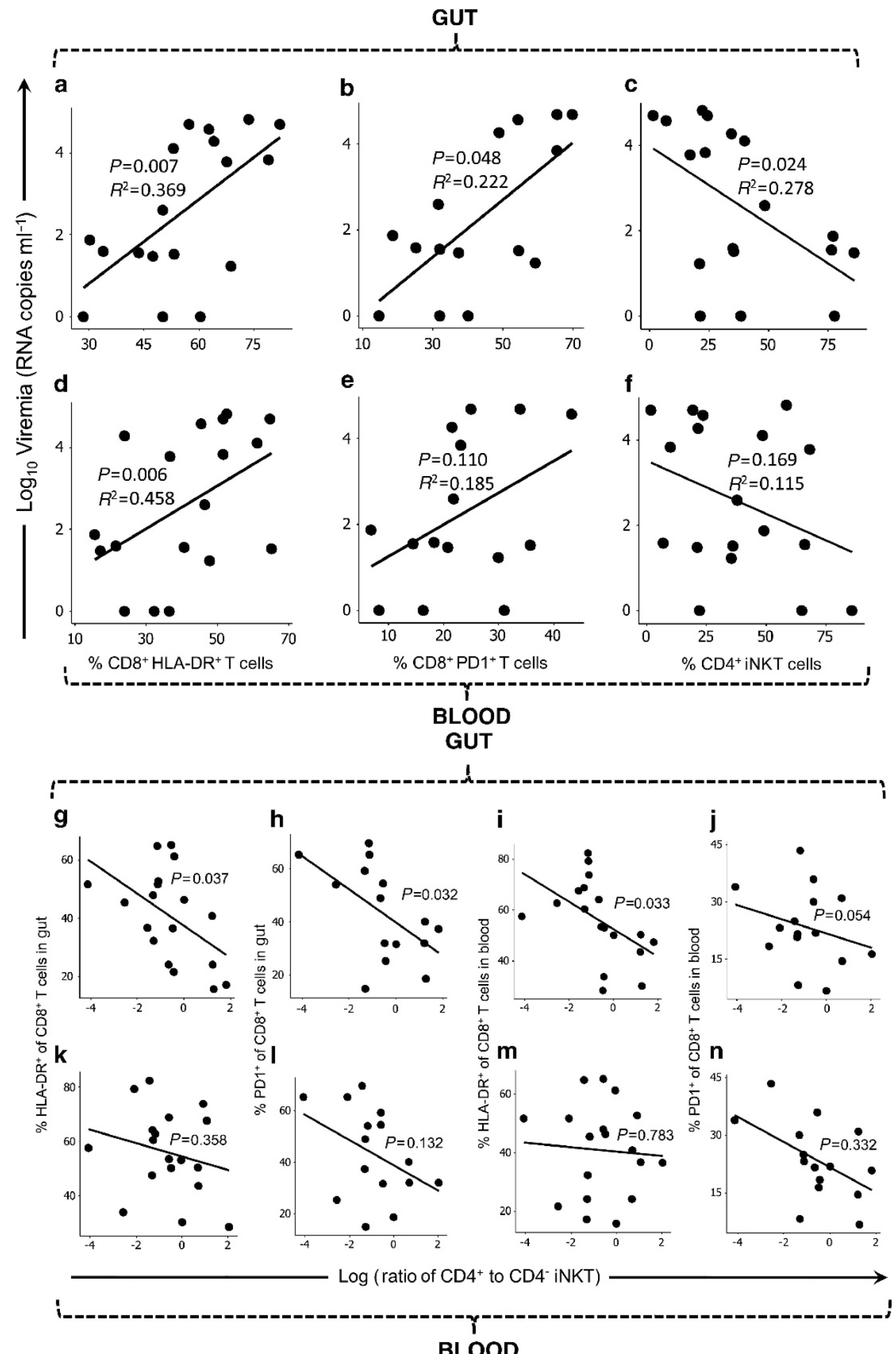

Figure 6 Correlations between the balance of $\mathrm{CD}^{+}$and $\mathrm{CD}^{-}{ }^{-}$iNKT cells (invariant natural killer T cells) to markers of immune activation and senescence in the blood and gut mucosal compartments of persons with HIV-1 infection. Plasma viremia of HIV-1-infected persons is plotted against the percentages of $\mathrm{CD}^{+} \mathrm{T}$ cells in the $(\mathbf{a}, \mathbf{b})$ gut mucosa and $(\mathbf{d}, \mathbf{e})$ blood expressing human leukocyte antigen (HLA)-DR $(\mathbf{a}, \mathbf{d})$ and programmed cell death-1 (PD-1) (b,e). Viremia is also plotted against the percentages of CD4 ${ }^{+}$iNKT cells in the (c) gut mucosa and (f) blood. The ratios of CD4 ${ }^{+}$to CD4 iNKT cells in the $(\mathbf{g}-\mathbf{j})$ gut mucosal and $(\mathbf{k}-\mathbf{n})$ blood compartments are plotted against the percentages of CD8 ${ }^{+} \mathrm{T}$ cells expressing HLA-DR in the gut $(\mathbf{g}$ $\mathbf{k}$ ) and blood (i, m) or PD-1 in the gut (h, I) and blood (j, n). There were insufficient cells for PD-1 analysis of Subjects 40729,49022 , and 49023.

translocation from the gut to peripheral blood, resulting in chronic systemic immune activation that drives ongoing loss of $\mathrm{CD}^{+} \mathrm{T}$ cells and disease progression in HIV-1 infection. ${ }^{24,25,32}$ Although iNKT cells have been observed in the human ${ }^{13,33,34}$ and murine ${ }^{35-37}$ gut and one study has demonstrated that dysregulation of gut iNKT cells in humans 
can lead to disease (ulcerative colitis), ${ }^{38}$ the impact of HIV-1 infection on this immune regulation in this compartment has been unexplored previously. Our data suggest that gut $\mathrm{CD} 4{ }^{+}$ iNKT cells are depleted by HIV-1 infection and suggest an alternative (but not mutually exclusive) mechanism of increased immune activation in the gut.

At baseline in healthy persons, we find that the total iNKT-cell frequency is similar between blood and gut, while the $\mathrm{CD} 4^{+}$ subset is more prominent in the gut. Given the anti-inflammatory immunomodulatory properties of these cells, this is consistent with a Th2 regulatory role of $\mathrm{CD} 4{ }^{+}$iNKT cells in moderating the vigorous inflammation in this compartment. In HIV-1-infected persons, there is a relative loss of the $\mathrm{CD}^{+}{ }^{+}$iNKT subset (particularly those expressing CCR5) that is most pronounced in the gut compartment, consistent with the vulnerability of these cells to HIV-1 infection. ${ }^{19}$ Although the remaining gut $\mathrm{CD} 4{ }^{+}$ iNKT cells retain the capacity to produce IL-4, imbalance of $\mathrm{CD}^{+}{ }^{+}$vs. $\mathrm{CD} 4^{-}{ }^{-}$iNKT cells is correlated to increased activation and exhaustion of the CD8 ${ }^{+} \mathrm{T}$ cells systemically. This suggests that there is inadequate compensatory anti-inflammatory activity by these key regulatory cells in the gut after HIV-1 infection and that the degree to which the balance of $\mathrm{CD} 4^{+}$vs. $\mathrm{CD} 4^{-}$iNKT cells is perturbed by HIV-1 infection in the gut (but not in the blood) is a key contributor to the inappropriate systemic immune activation driving HIV-1 pathogenesis.

A recent report regarding iNKT cells in Sooty Mangabeys lends additional support to this concept. ${ }^{39}$ Rout et al. studied these natural hosts for Simian Immunodeficiency Virus (SIV), who have asymptomatic chronic infection and maintain high peripheral blood total $\mathrm{CD} 4^{+} \mathrm{T}$-cell levels despite high viremia. These animals do not exhibit the immune activation associated with progressive $\mathrm{CD} 4{ }^{+} \mathrm{T}$-cell decline in the SIV-infected rhesus macaque model of AIDS. This study found that in contrast to rhesus macaques (and humans), Sooty Mangabeys lack CD4 ${ }^{+}$iNKT cells but instead mediate anti-inflammatory effects via release of IL- 10 by $\mathrm{CD} 4^{-}$ iNKT cells. Thus, there is no perturbation of iNKT cells by SIV infection compared with rhesus macaques and humans who have $\mathrm{CD}^{+}{ }^{+}$and $\mathrm{CCR}^{+}{ }^{+}$iNKT cells.

The Th17 axis has been implicated as being important for gut flora containment and suggested to be depressed in HIV-1 infection, resulting in microbial translocation and immune activation. ${ }^{40}$ Although murine data have suggested that early thymic emigrant iNKT cells can produce IL-1 $1{ }^{41}$ this has not been observed in humans. ${ }^{7}$ Our data substantiate activity of the Th17 axis in the bulk $\mathrm{CD}^{+}{ }^{+} \mathrm{T}$ cells of the gut compartment but not IL17 production by iNKT cells. It is unclear, however, whether iNKT cells might produce IL-17 under other experimental conditions. ${ }^{42}$ Moreover, while others have noted disruption of Th17 activity in the gut of persons with HIV-1 infection, ${ }^{40,43-46}$ we detect its reduction in the blood but not in the gut compartments. Methodological differences or lack of sufficient power with our cohort may account for this discrepancy.

In summary, our data address an underlying mechanism for the systemic immune activation that characterizes HIV-1 infection and mediates progression of immunodeficiency. We observe that preferential loss of the $\mathrm{CD} 4{ }^{+}{ }^{+}$iNKT cells in the gut but not in the blood compartment correlates with immune activation in both the gut and peripheral blood compartments, as measured by markers that are clinically predictive. Although it has been suggested that total $\mathrm{CD} 4^{+}$Th cell depletion in the gut drives microbial translocation and systemic immune activation, ${ }^{25,47}$ our results provide an alternative explanation by suggesting that the key underlying defect may be immune dysregulation within the gut itself. This does not exclude a role for microbial translocation in this process, but it is unclear whether translocation is primarily involved or an epiphenomenon related to the iNKT-cell imbalance-mediated immune dysregulation. Overall, our observations reinforce the central importance of the gut compartment in HIV-1 pathogenesis and demonstrate a key immune regulatory defect in this major reservoir of $\mathrm{T}$ cells.

\section{METHODS}

Subjects. Caucasian men were studied to minimize potential variation due to race and gender. The 25 participants included 7 control seronegative and 18 chronically HIV-1-infected men with selfreported to have peripheral blood $\mathrm{CD}^{+} \mathrm{T}$ lymphocyte counts $>300$ cells $\mu \mathrm{l}^{-1}$ without antiretroviral treatment for at least 6 months. Routine clinical laboratory testing of peripheral blood $\mathrm{CD}^{+}{ }^{+} \mathrm{T}$ cells and HIV-1 RNA was performed through the clinical laboratories at UCLA. All participants provided written informed consent under a University of California IRB-approved protocol. Demographics of these subjects are provided in Supplementary Table S1 online.

Mononuclear cell isolation. Peripheral blood mononuclear cells were isolated by Ficoll-Hypaque gradient centrifugation. Sigmoid colonic mucosal mononuclear cells were isolated as previously reported in detail. ${ }^{48,49}$ Briefly, 20 biopsies were obtained approximately $30 \mathrm{~cm}$ from the anal verge via flexible sigmoidoscopy using large-cup $(3.3 \mathrm{~mm})$ biopsy forceps. The biopsies were washed twice in RPMI 1640 medium with L-glutamine and HEPES (4-(2-hydroxyethyl)-1piperazineethanesulfonic acid), supplemented with $2.5 \mathrm{mg} \mathrm{ml}^{-1}$ of amphotericin B (Fungione, GIBCO Invitrogen, Carlsbad, CA) and $0.1 \mathrm{mg} \mathrm{ml}^{-1}$ of piperacillin-tazobactam (Zosyn, Lederle, Puerto Rico), and collagenase digested to obtain mononuclear cell suspensions, which were immediately stained and analyzed by flow cytometry. This typically yielded $2-5$ million viable $\mathrm{CD}^{+}{ }^{+} \mathrm{T}$ cells per 17 processed biopsy samples, as assessed by flow cytometry using Trucount (BD Biosciences, San Jose, CA). Cell viability was consistently above $90 \%$ as assessed by trypan blue exclusion.

Cell phenotyping by surface markers. Flow cytometry was performed on freshly isolated mononuclear cells. The iNKT-cell population was defined by staining $0.5-2.0 \times 10^{6}$ viable cells with saturating concentrations of anti-TCR V $\alpha 24$-phycoerythrin (PE), anti-TCR V $\beta 11$-fluorescein isothiocyanate (FITC), anti-CD3-peridinin chlorophyll protein (PerCP), anti-CD4-phycoerythrin-Cy7 (PECy7), and anti-CD8-allophycocyanin Cy-7 (APC-Cy7) for analysis on a FACSCanto flow cytometer (Becton Dickinson, San Jose, CA). Notably, staining for CD3, V $\alpha 24$, and V $\beta 11$ on lymphocytes has been shown to be highly sensitive and specific for iNKT cells, ${ }^{50}$ which we confirmed in control experiments demonstrating $90 \%$ specificity of iNKT-cell identification using a combination of the monoclonal antibody 6B11 (specific for the iNKT-cell receptor) in conjunction with staining for $\mathrm{CD} 3, \mathrm{~V} \alpha 24$, and $\mathrm{V} \beta 11$ (data not shown). Additional phenotyping was assessed by concomitant staining with allophycocyanin (APC)-labeled, anti-programmed cell death-1, anti-CCR5, or anti-HLA-DR antibodies. All monoclonal antibodies are delineated in Supplementary Table S2 online. Examples of the gating strategies are shown in Supplementary Figures S1 and 2. 
Assessment of cell function using intracellular cytokine analysis. Peripheral blood mononuclear cells or mucosal mononuclear cells were incubated for $4 \mathrm{~h}$ at $37^{\circ} \mathrm{C}$ in the presence of $25 \mathrm{ng} \mathrm{ml}{ }^{-1}$ of phorbol 12-myristate 13-acetate (Sigma, St Louis, MO), $1 \mu \mathrm{g} \mathrm{ml}^{-1}$ of ionomycin (Sigma), BD GolgiStop (BD Biosciences), and $10 \mu \mathrm{g} \mathrm{ml}^{-1}$ of Brefeldin A (Sigma). After two washes with phosphate-buffered saline containing $0.1 \%$ bovine serum albumin $(0.1 \%$ BSA-PBS), the cells were stained for $15 \mathrm{~min}$ at room temperature with anti-TCR V $\alpha 24-P E$, anti-TCR V $\beta 11-F I T C$, anti-CD3-PerCP, and anti-CD4- or anti-CD8- APC-Cy7. After a wash with 0.1\% BSA-PBS, the cells were fixated and permeabilized using the Cytofix/Cytoperm Plus kit as recommended by the manufacturer (BD Biosciences). Cells were then incubated for $20 \mathrm{~min}$ at room temperature with anti-gamma interferon-phycoerythrin-Cy7 plus either anti-IL-4- or anti IL-17- APC, followed by washing and immediate analysis by flow cytometry. A minimum of $3 \times 10^{5}$ cells per tube were analyzed. An example is show in Supplementary Figure S3.

Statistical analyses. At least $5 \times 10^{5} \mathrm{CD}^{+}$lymphocytes were collected through flow cytometry for all experiments. The nonparametric Mann-Whitney test was utilized for assessing differences between subpopulations. Pearson correlations were utilized for dot plots comparisons. Statistical analyses were carried out with Minitab Statistical Software (State College, PA). Statistical significance was defined as a $P$ value of $<0.05$ for the null hypothesis.

SUPPLEMENTARY MATERIAL is linked to the online version of the paper at http://www.nature.com/mi

\section{ACKNOWLEDGEMENTS}

This work was funded by NIH grant Al063974 (O.O.Y.) with assistance from the UCLA Mucosal Immunology Core Facility supported by the UCLA CFAR NIH grant Al028697.

\section{DISCLOSURE}

The authors declare no conflict of interest.

c) 2013 Society for Mucosal Immunology

\section{REFERENCES}

1. Wilson, S.B. \& Delovitch, T.L. Janus-like role of regulatory iNKT cells in autoimmune disease and tumour immunity. Nat. Rev. Immunol. 3, 211-222 (2003).

2. Godfrey, D.I., Stankovic, S. \& Baxter, A.G. Raising the NKT cell family. Nat. Immunol. 11, 197-206 (2010).

3. Godfrey, D.I. \& Rossjohn, J. New ways to turn on NKT cells. J. Exp. Med. 208, 1121-1125 (2011).

4. Joyce, S., Girardi, E. \& Zajonc, D.M. NKT cell ligand recognition logic: molecular basis for a synaptic duet and transmission of inflammatory effectors. J. Immunol. 187, 1081-1089 (2011).

5. Yang, O.O. et al. CD1d on myeloid dendritic cells stimulates cytokine secretion from and cytolytic activity of $V$ alpha $24 \mathrm{~J}$ alpha $Q T$ cells: a feedback mechanism for immune regulation. J. Immunol. 165, 3756-3762 (2000).

6. Brigl, M. et al. Innate and cytokine-driven signals, rather than microbial antigens, dominate in natural killer $\mathrm{T}$ cell activation during microbial infection. J. Exp. Med. 208, 1163-1177 (2011).

7. Snyder-Cappione, J.E. et al. A comprehensive ex vivo functional analysis of human NKT cells reveals production of MIP1-alpha and MIP1-beta, a lack of IL-17, and a Th1-bias in males. PLoS One 5, e15412 (2010).

8. Gumperz, J.E., Miyake, S., Yamamura, T. \& Brenner, M.B. Functionally distinct subsets of CD1d-restricted natural killer T cells revealed by CD1d tetramer staining. J. Exp. Med. 195, 625-636 (2002).

9. Wilson, S.B. et al. Extreme Th1 bias of invariant $V$ alpha $24 \mathrm{~J}$ alpha $Q \mathrm{~T}$ cells in type 1 diabetes. Nature 391, 177-181 (1998).

10. Brigl, M. \& Brenner, M.B. CD1: Antigen presentation and T cell function. Annu. Rev. Immunol. 22, 817-890 (2004).
11. Berzins, S.P., Smyth, M.J. \& Baxter, A.G. Presumed guilty: natural killer T cell defects and human disease. Nat. Rev. Immunol. 11, 131-142 (2011).

12. Lee, P.T., Benlagha, K., Teyton, L. \& Bendelac, A. Distinct functional lineages of human $V$ alpha-24 natural killer T cells. J. Exp. Med. 195, 637-641 (2002).

13. Fleuridor, R. et al. CD1d-restricted natural killer Tcells are potent targets for human immunodeficiency virus infection. Immunology 108, 3-9 (2003).

14. Thomas, S.Y. et al. CD1d-restricted NKT cells express a chemokine receptor profile indicative of Th1-type inflammatory homing cells. J. Immunol. 171, 2571-2580 (2003).

15. Motsinger, A. et al. CD1d-restricted human natural killer T cells are highly susceptible to human immunodeficiency virus 1 infection. J. Exp. Med. 195, 869-879 (2002)

16. Crowe, N.Y., Godfrey, D.I. \& Baxter, A.G. Natural killer T cells are targets for human immunodeficiency virus infection. Immunology 108, 1-2 (2003).

17. Li, D. \& Xu, X.N. NKT cells in HIV-1 infection. Cell Res. 18, 817-822 (2008).

18. van der $V$ liet, $H$.J. et al. Selective decrease in circulating $V$ alpha $24+V$ beta $11+$ NKT cells during HIV type 1 infection. J. Immunol. 168, 1490-1495 (2002).

19. Sandberg, J.K. et al. Selective loss of innate CD4 $(+) \vee$ alpha 24 natural killer $\mathrm{T}$ cells in human immunodeficiency virus infection. J. Virol. 76, 7528-7534 (2002).

20. Yang, O.O. et al. Delayed reconstitution of CD4 + iNKT cells after effective HIV type 1 therapy. AIDS Res. Hum. Retroviruses 23, 913-922 (2007).

21. Mowat, A.M. \& Viney, J.L. The anatomical basis of intestinal immunity. Immunol. Rev. 156, 145-166 (1997).

22. Anton, P.A. et al. Enhanced levels of functional HIV-1 co-receptors on human mucosal T cells demonstrated using intestinal biopsy tissue. AIDS 14, 1761-1765 (2000).

23. Shacklett, B.L. \& Anton, P.A. HIV infection and gut mucosal immune function: updates on pathogenesis with implications for management and intervention. Curr. Infect. Dis. Rep. 12, 19-27 (2010).

24. Schneider, T. et al. Loss of Cd4 T-lymphocytes in patients infected with human-immunodeficiency-virus type-1 is more pronounced in the duodenal mucosa than in the peripheral-blood. Gut 37, 524-529 (1995).

25. Brenchley, J.M. \& Douek, D.C. HIV infection and the gastrointestinal immune system. Mucosal Immunol. 1, 23-30 (2008).

26. Marchetti, G. et al. Microbial translocation predicts disease progression of HIV-infected antiretroviral-naive patients with high CD4 + cell count. AIDS 25, 1385-1394 (2011).

27. Merlini, E. et al. Evidence for polymicrobic flora translocating in peripheral blood of HIV-infected patients with poor immune response to antiretroviral therapy. PLoS One. 6, e18580 (2011).

28. Unutmaz, D. NKT cells and HIV infection. Microbes Infect. 5, 1041-1047 (2003).

29. Mothe, B. et al. CTL responses of high functional avidity and broad variant cross-reactivity are associated with HIV control. PLoS One. 7, e29717 (2012).

30. Grossman, Z., Meier-Schellersheim, M., Paul, W.E. \& Picker, L.J. Pathogenesis of HIV infection: what the virus spares is as important as what it destroys. Nat. Med. 12, 289-295 (2006).

31. Fevrier, M., Dorgham, K. \& Rebollo, A. CD4 + T cell depletion in human immunodeficiency virus (HIV) infection: role of apoptosis. Viruses 3 , 586-612 (2011).

32. Guadalupe, M. et al. Severe CD4(+) T-cell depletion in gut lymphoid tissue during primary human immunodeficiency virus type 1 infection and substantial delay in restoration following highly active antiretroviral therapy. J. Virol. 77, 11708-11717 (2003).

33. Van Dieren, J.M. et al. Roles of CD1d-restricted NKT cells in the intestine. Inflamm. Bowel Dis. 13, 1146-1152 (2007).

34. Kaser, A., Zeissig, S. \& Blumberg, R.S. Inflammatory bowel disease. Annu. Rev. Immunol. 28, 573-621 (2010).

35. Wei, B. et al. Commensal microbiota and CD8 + T cells shape the formation of invariant NKT cells. J. Immunol. 184, 1218-1226 (2010).

36. Middendorp, S. \& Nieuwenhuis, E.E. NKT cells in mucosal immunity. Mucosal Immunol. 2, 393-402 (2009).

37. Nieuwenhuis, E.E. et al. Cd1d-dependent regulation of bacterial colonization in the intestine of mice. J. Clin. Invest. 119, 1241-1250 (2009). 


\section{ARTICLES}

38. Fuss, I.J. et al. Nonclassical CD1d-restricted NKT cells that produce IL-13 characterize an atypical Th2 response in ulcerative colitis. J. Clin. Invest. 113, 1490-1497 (2004).

39. Rout, N. et al. Paucity of CD4 + natural killer T (NKT) lymphocytes in Sooty Mangabeys is associated with lack of NKT cell depletion after SIV infection. PLoS One. 5, e9787 (2010).

40. Salgado, M. et al. Long-term non-progressors display a greater number of Th17 cells than HIV-infected typical progressors. Clin. Immunol. 139, 110114 (2011).

41. Milpied, P. et al. IL-17-producing invariant NKT cells in lymphoid organs are recent thymic emigrants identified by neuropilin-1 expression. Blood 118 2993-3002 (2011).

42. Moreira-Teixeira, L. et al. Proinflammatory environment dictates the IL-17producing capacity of human invariant NKT cells. J. Immunol. 186, 57585765 (2011).

43. Prendergast, A. et al. HIV-1 infection is characterized by profound depletion of CD161 + Th17 cells and gradual decline in regulatory T cells. AIDS 24, 491-502 (2010).
44. Elhed, A. \& Unutmaz, D. Th17 cells and HIV infection. Curr. Opin. HIV AIDS 5, 146-150 (2010).

45. El, H.A. et al. Susceptibility of human Th17 cells to human immunodeficiency virus and their perturbation during infection. J. Infect. Dis. 201, 843-854 (2010).

46. Ndhlovu, L.C. et al. Suppression of HIV-1 plasma viral load below detection preserves IL-17 producing T cells in HIV-1 infection. AIDS 22, 990-992 (2008).

47. Haynes, B.F. Gut microbes out of control in HIV infection. Nat. Med. 12, 1351-1352 (2006).

48. Shacklett, B.L. et al. Optimization of methods to assess human mucosal T-cell responses to HIV infection. J. Immunol. Methods 279, 17-31 (2003).

49. Ibarrondo, F.J. et al. Parallel human immunodeficiency virus type 1-specific CD8 + T-lymphocyte responses in blood and mucosa during chronic infection. J. Virol. 79, 4289-4297 (2005).

50. Bricard, G. et al. Enrichment of human CD4 + V(alpha)24/Vbeta11 invariant NKT cells in intrahepatic malignant tumors. J. Immunol. 182, 5140-5151 (2009). 